\title{
Ultrasonic monitoring of dentin demineralization
}

\author{
Josep Rodríguez-Sendra, Inés Torres, Noé Jiménez, Salvatore Sauro, Francisco Camarena
}

\begin{abstract}
Demineralization is a process of loss of minerals in the dental hard tissue that affects seriously the health of the patients, as it diminishes the tooth resistance, generating chewing problems by altering the occlusal structure, hypersensitivity and pulpal problems. Demineralization can be produced by pathological processes as erosion or caries, or by surgical processes as etching. Due to the complexity of natural demineralization processes, it is mandatory to provide quantitative and standardized tests to allow their study in controlled laboratory conditions. Ultrasonic techniques are suitable for this purpose as they are non-destructive, quick and provide localized mechanical information about the tissue, which is related with its degree of demineralization. In the present work we evaluate the complete process of demineralization of the human dentin under controlled laboratory conditions using a pulse-echo ultrasonic technique. Up to 15 human dentin teeth have been demineralized with phosphoric acid at $10 \%$. The time-of-flight measurements using the pulse-echo system allows to obtain the speed of sound in healthy $(3415 \mathrm{~m} / \mathrm{s})$ and demineralized dentin tissue $(1710 \mathrm{~m} / \mathrm{s})$, as well as to characterize the dynamical process of the acid penetration, which generates well-defined boundaries between two media (demineralized and mineralized dentin), showing very different mechanical properties. These boundaries advance in depth at an initial rate of $2.9 \mu \mathrm{m} / \mathrm{min}$, decelerating at $9.3 \mathrm{~nm} / \mathrm{min}^{2}$ until the whole demineralization of the sample is achieved. In addition, the technique allows to measure the relevance of the demineralization produced by the acid residues inside the tooth once it has been removed from the acidic solution. Beyond the assessment of artificial demineralization lesions under laboratory conditions, as demonstrated in this paper, the proposed technique opens new approaches to the assessment of demineralization caused by natural caries in vivo.
\end{abstract}

Index Terms-Denistry; Demineralization; Ultrasonic; Pulseecho.

\section{INTRODUCTION}

D ENTAL caries is one of the most prevalent diseases worldwide. According to the World Health Organization, between $60 \%$ and $90 \%$ of school-age children and almost $100 \%$ of adults have or have had caries injuries [1]. Dental caries is a multifactorial disease that appears when, in a first stage, enamel and, then, dentin lose their minerals due to acids produced from bacterial metabolism when break down sugars or food debris [2], [3]. Knowledge of the demineralization process in dentin is crucial in cavity preparation, as the dentist must remove part of the dentin to place a filling and the criterion for deciding when to stop the removal of dentin material has to do with its degree of mineralization. Objective criteria should be established at this point. On the other hand, the use of bioactive materials has recently been proposed to facilitate the remineralization of damaged dentin, making it possible to preserve a greater amount of patient's dentin [4][6]. Thus, it is important to be able to assess the degree of mineralization of the dentin to make these decisions and to be able to quantify the degree of improvement of the new products.

Dentin consists of about $70 \%$ inorganic material (hydroxyapatite), $20 \%$ organic base (highly mineralized type I collagen fibers) and about $10 \%$ water [7]. Its demineralization process involves at least two stages: the dissolution of biominerals by organic acids and the subsequent degradation of dentin matrix by proteases [8]. Natural dentin demineralization processes are geometrically complex, with variations in width, depth, severity and color, making it difficult to find many similar lesions for in vitro studies [8]. For this reason, standardized artificial protocols using acids have been proposed in order to reproduce real lesions and more meticulous investigations with the combination of new technologies are required [8].

Artificial standardized demineralization lesions offer many advantages compared to the use of natural lesions for research because demineralization protocols can be reproducible and lesions with different size can be readily made based on demineralization kinetics of a given acid [9]. Some studies have shown that these acids, mainly ethylenediaminetetraacetic acid (EDTA) or phosphoric acid (PA), can selectively demineralize extrafibrillar minerals and keep intrafibrillar minerals at least partially, if not completely intact, both of them demineralizing with minimum collagen degradation [10]. The acidic agents remove the smear layer and open the dentin tubules, demineralize the dentin surface and increase the porosity of the intertubular dentin [11].

Different techniques have been proposed to study the demineralization process of the tooth, such as Scanning Electron Microscopy (SEM) [12], Atomic Force Microscopy (AFM) [13], X-ray tomographic microscopy (XTM) [14], Optical Coherence Tomography (OCT) [15] or nanoindentation [16]. Attin et al. described in 2014 different techniques and methods for assessment the dental erosion [17].

Ultrasonics techniques have also been widely used for tooth characterization during last decades. In 1963 Baum et al. used ultrasound to image the internal structures of the tooth [18]. In 2008 Ghorayeb et al. presented a review which examined the diagnostic applications of dental ultrasound, starting with the pioneering work up to the current work [19]. In addition, Marotti et al. presented in 2013 a review where it was described and discussed the use of ultrasound in several areas as dental scanning, caries detection, and implant dentinstry among others [20].

The acoustic properties of dental tissues have been evaluated using non-invasive ultrasonic techniques by different authors. Watanabe et al. measured the longitudinal and shear sound velocity to determine the elastic modulus in mineralized (17.4 $\mathrm{GPa}$ ) and demineralized (1.46 GPa) human dentin, finding big differences [21]. A similar study was performed by Yasuda et al. for bovine tooth, obtaining an elastic modulus of 16.9 

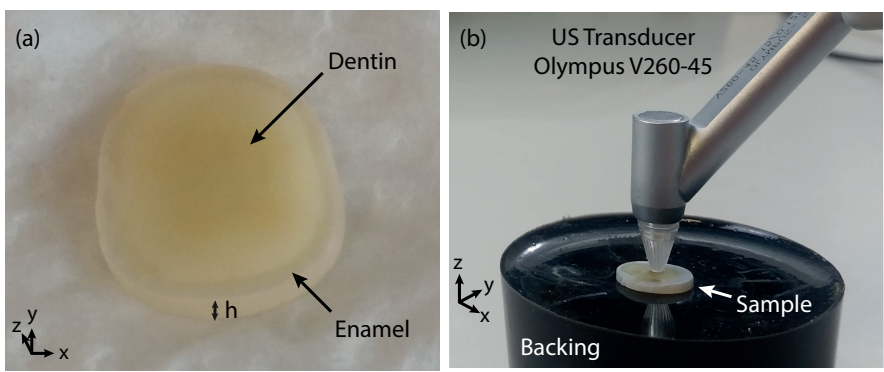

GPa (mineralized) and 2.1 GPa (demineralized) and a velocity of $3675 \mathrm{~m} / \mathrm{s}$ (mineralized) and $1597 \mathrm{~m} / \mathrm{s}$ (demineralized) [22]. These studies quantitatively show that dentin behaves close to a soft tissue after complete demineralization. Different authors measured longitudinal wave velocity in human molar dentin obtaining values from 3317 to $4050 \mathrm{~m} / \mathrm{s}$ as a function of the type of teeth [23]-[28]. C. Löst and C. John described a method which allows measurements on coplanar ground sections of human teeth with a lateral resolution of $0.2 \mathrm{~mm}$ using a water tank using a 20-MHz transducer [29], [30]. Miyazaki et al. showed that mechanical properties of tissue do not vary as long as the samples are taken with the same section of the same tooth [26]. Lees et al. used an ultrasonic system to continuously monitor the development of a demineralized layer due to etching by hydrochloric acid [31]. Yamaguchi et al. conducted an experiment in bovine tooth to demonstrate the protection capabilities against demineralization of the casein phosphopeptide-Amorphous calcium phosphate paste and the results were monitored comparing changes in the longitudinal wave velocity [32], [33]. A similar study was performed by Endo et al. to evaluate the apparently good protection capabilities of calcium phosphate desensitizer to prevent demineralization [34]. All these studies have demonstrated the hypothesis that the volumetric concentration of minerals in dental hard tissue is related with the propagation velocity of ultrasonic waves.

In the present work we study the complete process of demineralization of the human dentin under controlled laboratory conditions using a non-destructive pulse-echo ultrasonic technique. The main objective is to demonstrate the capability of ultrasound to monitor an artificial demineralization process of the dentin with acid, that is a widespread practice in dentistry to prepare samples for subsequent studies. The measurement of the time-of-flight of the acoustic pulses allows us to obtain the effective sound velocity in demineralized and healthy dentin tissue, as well as to demonstrate that the acid-penetration process defines a clear boundary between two media with very different mechanical properties. The present ultrasonic technique, which incorporates an adapter to reduce the contact area between the emitter and the dentin, is suitable to be used in in-vivo studies.

The paper is organized as follows: section II shows the sample preparation procedure, the demineralization protocol and the ultrasonic monitoring methods. Section III presents the results obtained for two demineralization studies. Finally, section IV shows the discussion and conclusions.

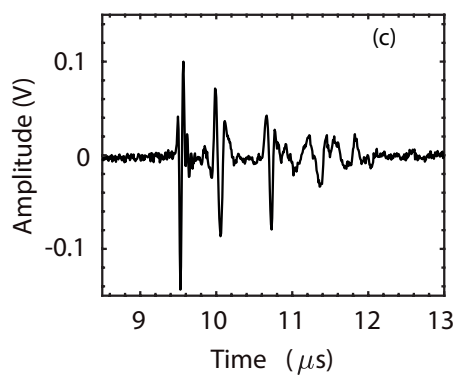

Fig. 1. (a) Photograph of a sample after preparation. (b) Photograph of the experimental set-up and (c) Example of a RF-signal obtained for a partially demineralized sample, showing several echoes.

\section{MATERIALS AND METHODS}

\section{A. Sample preparation}

A set of 18 healthy permanent human molars without caries or fillings extracted for orthodontic or periodontal reasons were obtained with informed consent. The teeth were stored in distilled water. The occlusal surfaces were polished using sequentially finer grades of polishing paper SI-C, grit 320, 500 and 1000 (Versocit, Struers A/S, Copenhagen, Denmark) until the occlusal enamel were completely removed. A polishing head that apply a constant force of $10 \mathrm{~N}$ (Vector LC Power Head, Buehler, Lake Bluff, Illinois, USA) in conjunction with a water cooled rotating polishing machine (Meta-Serv 3000 Grinder-Polisher, Buehler, Lake Bluff, Illinois, USA) was used at a rotating speed of 250 rotations per minute. The molars without occlusal surfaces were placed in a watercooled diamond saw (Micromet evolution, Remet, Bologna, Italy) and were cross sectioned to approximately 1.1-mm thick samples, as shown in Fig. 1 (a). The surfaces were polished with abrasive paper to obtain a standardized thickness of $1.09 \pm 0.05 \mathrm{~mm}$. All samples were immersed in distilled water and cleaned using an ultrasonic bath to remove the debris. After that, the samples were stored in distilled water.

\section{B. Demineralization procedure}

A total of 14 samples were located together with phosphoric acid (Panreac Química, Barcelona, Spain) at 10\% concentration in a Pyrex recipient and agitated with a magnetic shaker during the whole demineralization process.

A set of 6 samples were used for the SEM and Fourier Transform Infrared Spectroscopy (FTIR) evaluation. Half of the specimens, $N=3$, were completely demineralized for 5 hours and half of the specimens, $N=3$, were maintained in distilled water and used as control samples.

For the first ultrasonic experiment (E1), 5 samples were completely demineralized for 290 minutes and 1 sample was maintained in distilled water to be used as the baseline control. Every 10 minutes, all samples were removed from the acidic solution and cleaned with distilled water to perform the ultrasonic measurements, which delayed 3 minutes approximately. After that, the samples were returned to the acidic solution to continue with the demineralization process. The control sample was monitored following the same procedure but it was conserved in distilled water all the time.

For the second ultrasonic experiment (E2), 6 samples were demineralized to a different degree. The 6 samples were located in the Pyrex recipient with the phosphoric acid at $10 \%$ concentration. Each hour, one of the samples was extracted, 
cleaned, and ultrasonically monitored. Then, the sample was conserved in distilled water and monitored each 60 minutes.

\section{SEM and FTIR measurement}

First, 3 further sound samples and 3 demineralized samples were analyzed using ATR/FTIR Spectrometer (Perkin-Elmer, Beaconsfield, UK) with a spectral resolution of $4 \mathrm{~cm}^{-1}$ to characterize the chemical composition of the dentin before and after acid treatment. After the FTIR analysis, the samples were analyzed by SEM. The specimens were dehydrated in ascending concentration of alcohol, mounted on aluminum stubs, and sputter coated. The morphology of the specimens was analyzed using a Hitachi S3500 scanning electron microscope (Hitachi High Technologies, Maidenhead, UK).

\section{Ultrasonic measurement}

An Olympus SONOPEN V260-45 transducer with a nominal central frequency of $15 \mathrm{MHz}$ and a bandwidth from 5.98 to $17.68 \mathrm{MHz}(-6 \mathrm{~dB})$ was used as emitter and receiver using a pulse-echo technique to obtain the time of flight. This transducer presents a delay line with a diameter of $D=2 \mathrm{~mm}$ and a ratio between the diameter and the wavelength of $D / \lambda=20$ wavelengths per aperture in water. The choice of the source is a compromise between a small physical dimension mandatory for dental applications and a high $D / \lambda$ ratio to enhance the acoustic energy collected by the transducer.

Fig. 1 (b) show a photograph of the experimental setup. The transducer was placed perpendicularly to the top surface of the dentin sample to record the echo signals. At the opposite side, the sample was in contact with a rigid support to enhance the reflection of the ultrasonic waves at the boundary. An ultrasonic pulser-receiver (US-Wave, Lecoeur Electronique) was used to emit and receive ultrasonic pulses. The received signal was digitized within the pulser-receiver at a sampling frequency of $125 \mathrm{MHz}$. An example of the RFsignal registered for a partially demineralized sample is shown in Fig. 1 (c). For each experiment, a total of 500 acquisitions were performed in 5 different points on the top surface of the dentin (100 acquisitions per point, each point randomly distributed in the dentin area of each sample, see Fig. 1 (b)). The approximate duration of each ultrasonic measurement was 3 minutes. After that, the sample was returned to the acidic solution (ultrasonic experiment E1) or to the distilled water tank (ultrasonic experiment E2). All experiments were performed at a controlled temperature of $23 \pm 0.2^{\circ} \mathrm{C}$.

\section{E. Simulations}

Finite Element Method (FEM) simulations of the pulse propagation through layered and homogeneous media were performed using the Acoustics Module of COMSOL Multiphysics software in one dimension and in time domain. The experimental data measured by ultrasound methods before demineralization and after complete demineralization was used to define the mechanical properties of the demineralized and healthy dentin in the simulation. The input waveform in the simulations at the air-dentin boundary was a the first derivative of a Gaussian pulse (bipolar pulse) with spectral components from 5.98 to $17.68 \mathrm{MHz}$, i.e., similar to the experiments.

\section{RESULTS}

\section{A. SEM and FTIR experiments}

SEM examination reveals minerals loss due to the effect of the acid, as shown in Figs. 2 (a, b). After demineralization, the dentinal tubules are wider (compare Fig. 2 (b) and Fig. 2 (a)).

In the FTIR spectra, shown in Figs. 2 (c, d), the organic spectrum of the dentin (group Amide) is mostly preserved. Amide I and II show similar values before and after the acid bath. At the inorganic spectrum, there is an important loss of calcium-phosphate group (PO). Peaks of minerals at 961 and $1019 \mathrm{~cm}^{-1}$ that can be clearly observed in the mineralized case, shown in Fig. 2 (c), are hardly visible after the demineralization process caused by the acid, shown in Fig. 2 (d).

\section{B. Ultrasonic experiment 1}

Figure 3 (a) shows 29 RF-signals acquired during the ultrasonic monitoring of the demineralization process of one of the 5 samples during the first ultrasonic experiment (E1). The time between each measurement was 10 minutes during the demineralization process, reaching the complete demineralization after 280 minutes of acid action in this particular case. The RFsignals for the rest of the samples and for the control sample are shown in Figs. 4 (a-f). By inspecting the back-scattered signals in Fig. 3 (a) we observe that dentin can be considered as a homogeneous medium for the frequencies involved in these experiments. This follows from the absence of scatters in the first RF-signal ( $t=0 \mathrm{~min}$, corresponding to mineralized dentin) and in the last RF-signal ( $t=290 \mathrm{~min}$, corresponding to completely demineralized dentin). In the initial and final cases, the echoes correspond to the acoustic impedance change between the ultrasonic transducer and the top surface of the dentin (first echo) and between the dentin and the surface of black solid backing in Fig. 1 (b), (last echo). By measuring the sample thickness, the longitudinal wave velocity can be calculated for both, healthy dentin $(3415 \pm 30) \mathrm{m} / \mathrm{s}$ and completely demineralized dentin $(1710 \pm 70) \mathrm{m} / \mathrm{s}$ using the well-known relation

$$
c=\frac{2 h}{\Delta t},
$$

where $c$ is the longitudinal wave velocity in $\mathrm{m} / \mathrm{s}, h$ is the sample thickness in $\mathrm{m}$ and $\Delta t$ is the time between echoes in seconds, i.e., the time of flight. RF signals were processed using MATLAB (Mathworks, USA) to estimate the time delay of the echoes. First, the envelope of each signal was calculated as the absolute value of its Hilbert transform. Then, a peakdetector algorithm was used to estimate the time corresponding to the arrival time of the first two echoes. Finally, time of flight was calculated as the difference of these arrival times.

In contrast, clear echoes from well-defined boundaries can be observed during the demineralization process. Once the demineralization process starts (signals for $t>0 \mathrm{~min}$ ), two new clear echoes appear at the middle of the RF-signals, showing that there are two new boundaries inside the dentin. As demineralization time increases, the time of arrival of these echoes progressively changes as a consequence of the action of the acid on the dentin. A simple model to explain the way 

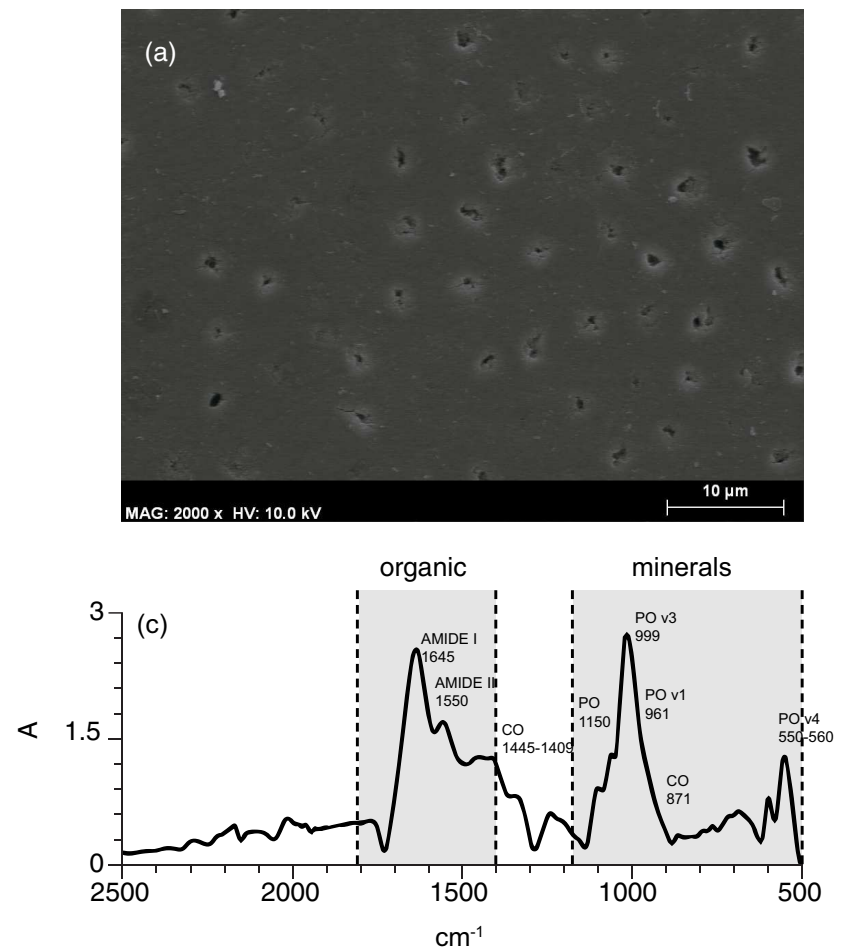
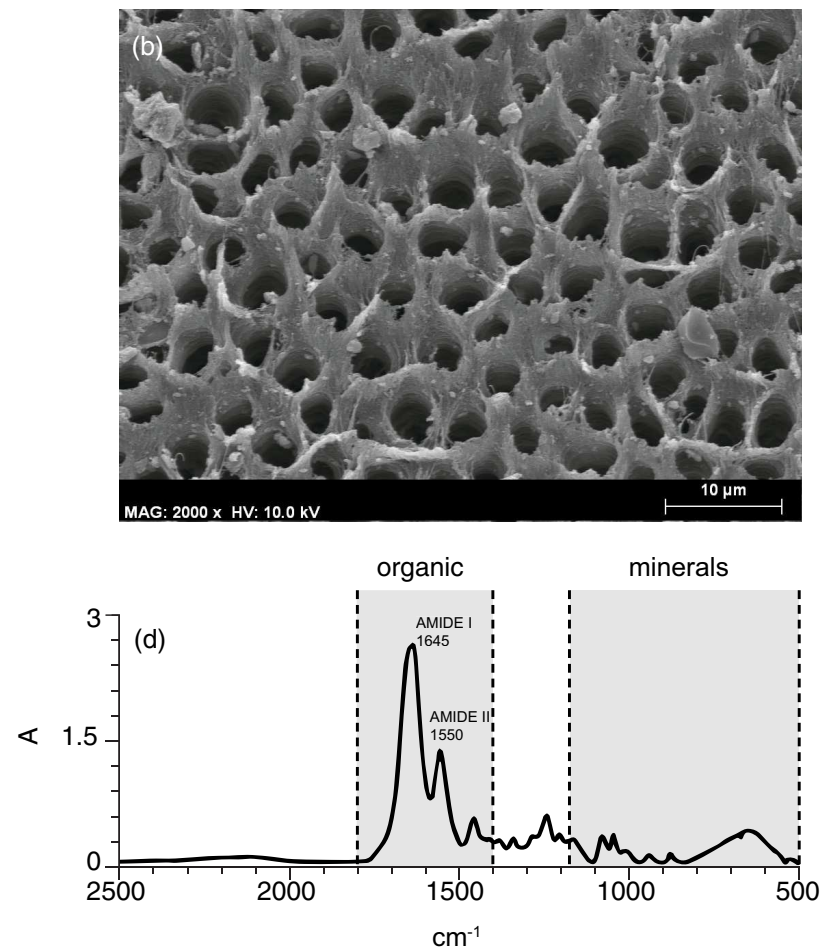

Fig. 2. Scanning Electron Microscopy (SEM) image of (a) a non-demineralized sample and (b) a demineralized sample. Fourier Transform Infrared Spectroscopy (FTIR) spectrum of (c) a non-demineralized sample and (d) a demineralized sample.

pulse-echo signals are changing is sketched in Figs. 3 (b). Here, we hypothesize that the action of the acid during the demineralization process generates a layered medium due to the progressive penetration and action of the acid towards the central core of the sample. As the acid acts from the top and bottom surfaces of the disk-shaped samples, the mechanical properties of the sample present a layered structure and at its well-defined boundaries echoes are produced. As the process evolves, the central layer becomes thinner and, eventually, all the sample is demineralized and the inner boundaries vanish, as observed in the RF-signals of Fig. 3 (a) at $t=290$ min. The whole process produces the characteristic pattern of asymmetric "W" that can be seen for all experimental samples in Fig. 4 (a-e). In contrast, during the ultrasonic monitoring of the control sample conserved in distilled water, shown in Fig. 4 (f), no internal echoes appeared.

Summarizing, a first echo at about $9.4 \mu \mathrm{s}$ is always visible for all experiments because it corresponds to the boundary between the delay line of the transducer and the top surface of the dentin, so it is not affected by the demineralization process. A second echo corresponds to the boundary between the first demineralized area and the central mineralized core of the dentin: the temporal delay of this echo increases during the experiment because the boundary is moving deeper in the dentin and the speed of sound in the demineralized layer decreases as the acid acts. The third echo corresponds to the boundary created by the acid etching from the bottom surface of the sample. The delay time of this echo is slightly reduced as acid acts (for some samples it almost remains constant) because the joint action of two opposite effects: on the one hand, the inner boundary gets closer to the ultrasonic probe and, on the other hand, the speed of sound in the demineralized layer is decreased. Finally, a fourth echo is generated at the bottom surface: it is progressively delayed because the demineralized layers of dentin with lower speed of sound grow during the demineralization process, consequently increasing the total time-of-flight of the reflected wave at this boundary.

Figure 4 shows in gray scale the amplitude of the echoes for the rest of samples. The last echo is attenuated once the acid creates the two internal boundaries which reflect an important part of the pulse energy to the ultrasonic probe. Only when the demineralization process is completed and the two internal boundaries disappear, the amplitude of the last echo increases again. In addition, note that at the end of the experiments the second and third echoes simultaneously vanish for all samples at a time that corresponds with the center of the sample, i.e., between the first and fourth echoes, showing a symmetric action of the acid from both sides of the sample.

The demineralized distance, $d(t)$, defined as the depth of

TABLE I

SAMPLE THICKNESS AND FITTED PARAMETERS.

\begin{tabular}{lccc}
\hline & $\begin{array}{c}\text { Thickness } \\
h(\mathrm{~mm})\end{array}$ & $\begin{array}{c}\text { Initial velocity } \\
v_{0}(\mu \mathrm{m} / \mathrm{min})\end{array}$ & $\begin{array}{c}\text { Acceleration } \\
a_{d}\left(\mathrm{~nm} / \mathrm{min}^{2}\right)\end{array}$ \\
\hline Sample 1 (D1) & 1.15 & $4.2 \pm 0.2$ & $-17.5 \pm 1.1$ \\
Sample 2 (D2) & 1.15 & $3.2 \pm 0.2$ & $-8.5 \pm 1.1$ \\
Sample 3 (D3) & 1.15 & $3.7 \pm 0.2$ & $-11.9 \pm 1.1$ \\
Sample 4 (D4) & 1.20 & $3.3 \pm 0.2$ & $-9.6 \pm 1.1$ \\
Sample 5 (D5) & 1.15 & $3.1 \pm 0.2$ & $-7.6 \pm 1.1$ \\
Mean & 1.16 & $3.5 \pm 0.4$ & $-11.0 \pm 3.0$ \\
\hline
\end{tabular}



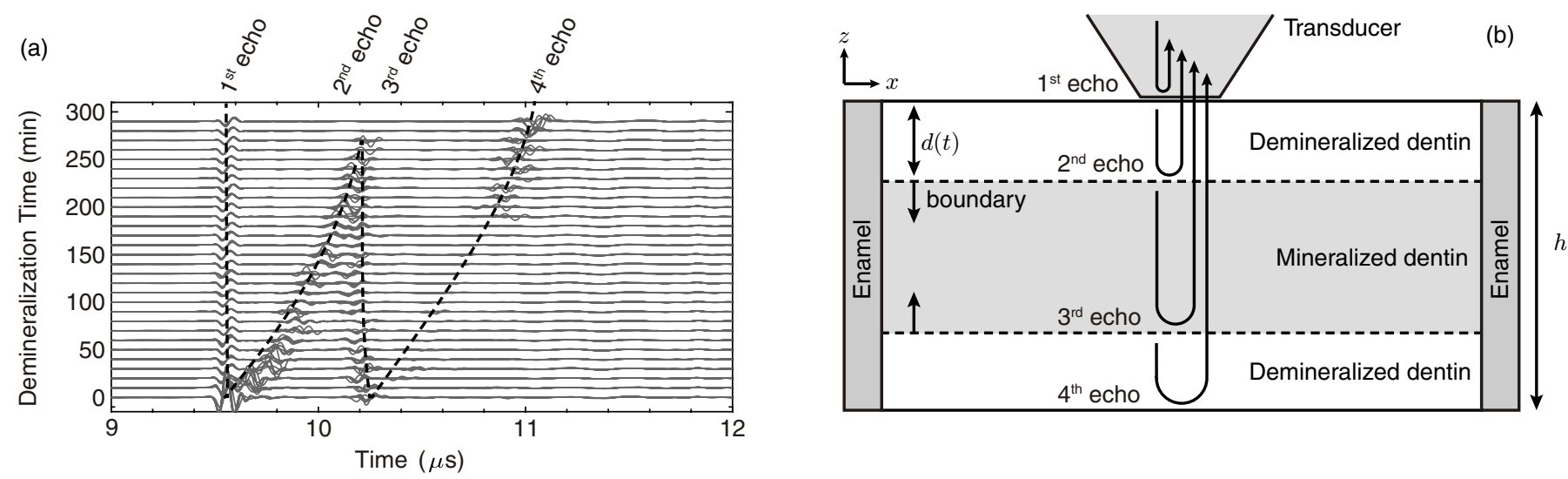

Fig. 3. (a) RF-signals corresponding to the ultrasonic monitoring of the whole demineralization process of sample D1. The dashed lines were drawn manually as an eye-guide. (b) Scheme of the layered dentin with different stiffness and the expected ultrasonic echoes according to the hypothesis that the acid effect creates well-defined boundaries. The depth of the demineralized-moving layer is marked as $d(t)$.

the demineralized layer, see Fig. 3 (b), is calculated from the time of flight between the first and second echoes assuming the speed of sound of the demineralized layer matches the value of the whole demineralized sample. The speed of sound in the demineralized medium is calculated from the last measurements using Eq. (1). Figure 5 (a-e) shows the demineralized distance as a function of demineralization time for the 5 samples studied. In addition, Fig. 5 (f) shows the results of the 5 samples together to demonstrate the good repeatability of the experiments. The demineralization presents a non uniform rate that decreases with time as a result of the natural process of diffusion of the acid in the dentin: the action of the acid is more efficient in the most superficial areas. As expected, at the end of the process the demineralization distance converge to half the sample thickness, $h / 2$, showing
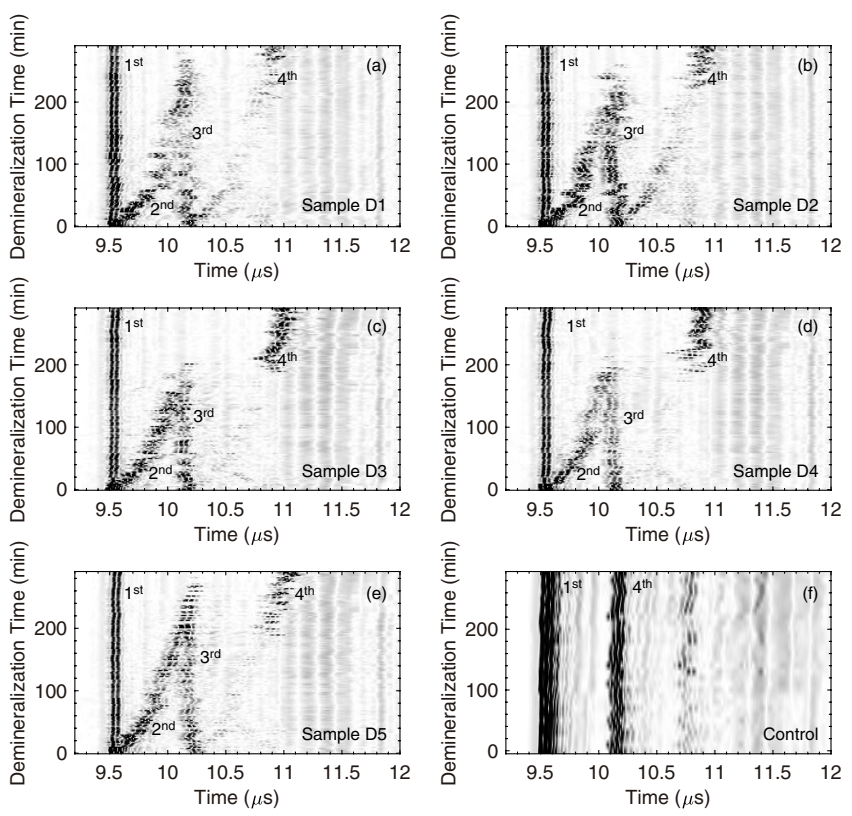

Fig. 4. (a-e) RF-signals, equivalent to single-element M-mode images, corresponding to the ultrasonic monitoring of the whole demineralization process of samples D1-D5 and (f) control sample. the complete demineralization of the samples.

A parabolic empirical model is fit to the experimental data to model the rate of demineralization as

$$
d(t)=v_{0} t+\frac{1}{2} a_{d} t^{2}
$$

where $d(t)$ is the demineralized distance, $v_{0}$ is the initial velocity of the demineralization process, $a_{d}$ is the acceleration of the demineralization process, and $t$ is the demineralization time.

The fitted coefficients for the 5 demineralized samples are listed in Table I. A good repeatability between experiments is observed. The results show that phosphoric acid at $10 \%$ concentration produces a mean initial demineralization velocity in human dentin of $v_{0}=3.5 \pm 0.4 \mu \mathrm{m} / \mathrm{min}$, with a mean deceleration of $a_{d}=-11.0 \pm 3.5 \mathrm{~nm} / \mathrm{min}^{2}$.

In order to further test the proposed hypothesis of the layered medium, a 1D FEM numerical acoustic simulation using a medium with three homogeneous layers was performed. The external layers were set as demineralized dentin $(c=1710$ $\mathrm{m} / \mathrm{s}$ ), and the central core was set to mineralized dentin $(c=3415 \mathrm{~m} / \mathrm{s})$. Two set of 200 simulations were performed moving the boundaries between the external and internal layers accordingly to the empirical model of all merged data $\left(v_{0}=3.5 \mu \mathrm{m} / \mathrm{min}, a_{d}=-11.0 \mathrm{~nm} / \mathrm{m}^{2}\right)$. For the first set of simulations, shown in Fig. 6 (a), the density was the same in both layers, corresponding to healthy dentin $(\rho=2010$ $\mathrm{kg} / \mathrm{m}^{3}$ ), and for the second set, shown in Fig. 6 (b), the density for the mineralized density was $\rho=2010 \mathrm{~kg} / \mathrm{m}^{3}$ and for the demineralized layer was $\rho=1050 \mathrm{~kg} / \mathrm{m}^{3}$ as reported in the literature [35].

The simulated RF-signals are shown in gray scale in Fig. 6 superimposed to the RF-signals measured experimentally for sample D1 (continuous lines). First, for the homogeneous density simulation (Fig. 6 (a)), it can be observed that the time of arrival of the simulated echoes matches the experimental one, as well as their evolution during the demineralization process (note signals were aligned at $t=0$ by delaying experimental data $9.4 \mu \mathrm{s}$ ). The fourth received echo is identified in the simulations as the one from the bottom boundary, while the 

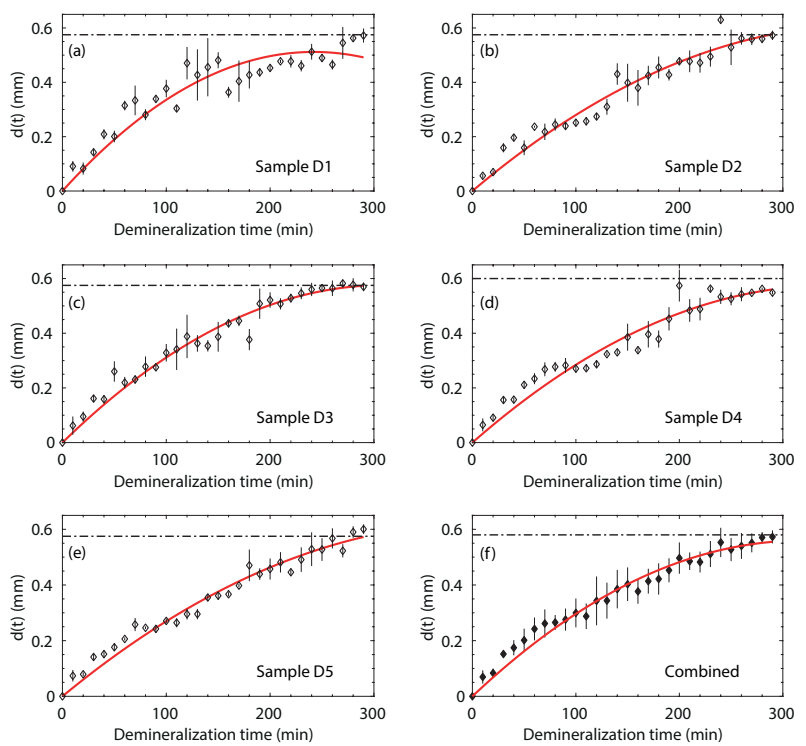

Fig. 5. (a-e) Demineralized distance versus demineralization time for samples D1-D5, respectively. Experimental data (markers) and parabolic empirical model (continuous red line). The dashed-dotted line shows half the thickness of each sample $(h / 2)$, i.e., the total demineralization state. (f) All data merged and corresponding fit.

second and third correspond to the inner boundaries. However, the impedance mismatch by only changing the sound speed in the simulation is weak and, therefore, a great amount of energy impinges the bottom boundary.

Figures 6 (b-e) shows the results of a more realistic simulation by setting different values for the density of the mineralized and demineralized dentin layers. It is clearly observed the screening effect of the inner layers which decreases the energy of the echoes reflected at the bottom boundary. When the inner dentin layer is very thin or it vanish due to a completely demineralization $(t>240 \mathrm{~min})$, the amplitude of the echo generated at the bottom boundary is strongly enhanced because the pulse is not scattered at any abrupt impedance change during the propagation and, therefore, it arrives at that boundary with greater energy. The dynamical processes observed in the simulations are in excellent agreement with all measured samples. In addition, a set of secondary echoes corresponding to second-order reflections are clearly visible in the simulated data $(t>1.2 \mu \mathrm{s})$ because the lack of absorption in the simulation. These secondary echoes do not interfere with the main echoes as they reach the ultrasonic probe later in time. Note that some secondary echoes can also be observed in the experimental data at the beginning of the demineralization process, in a roughly agreement with the simulation.

\section{Ultrasonic experiment 2}

A second experiment was designed to evaluate the inertia of the demineralizing effect of the phosphoric acid, i.e. the continuation of the demineralization process even when the dentin has been removed from the acidic solution. A set of 6 dentin samples (mean thickness $1.03 \pm 0.05 \mathrm{~mm}$ ) were demineralized following the procedure previously defined in Sec. II-B. Every hour, one sample was extracted from the (a)
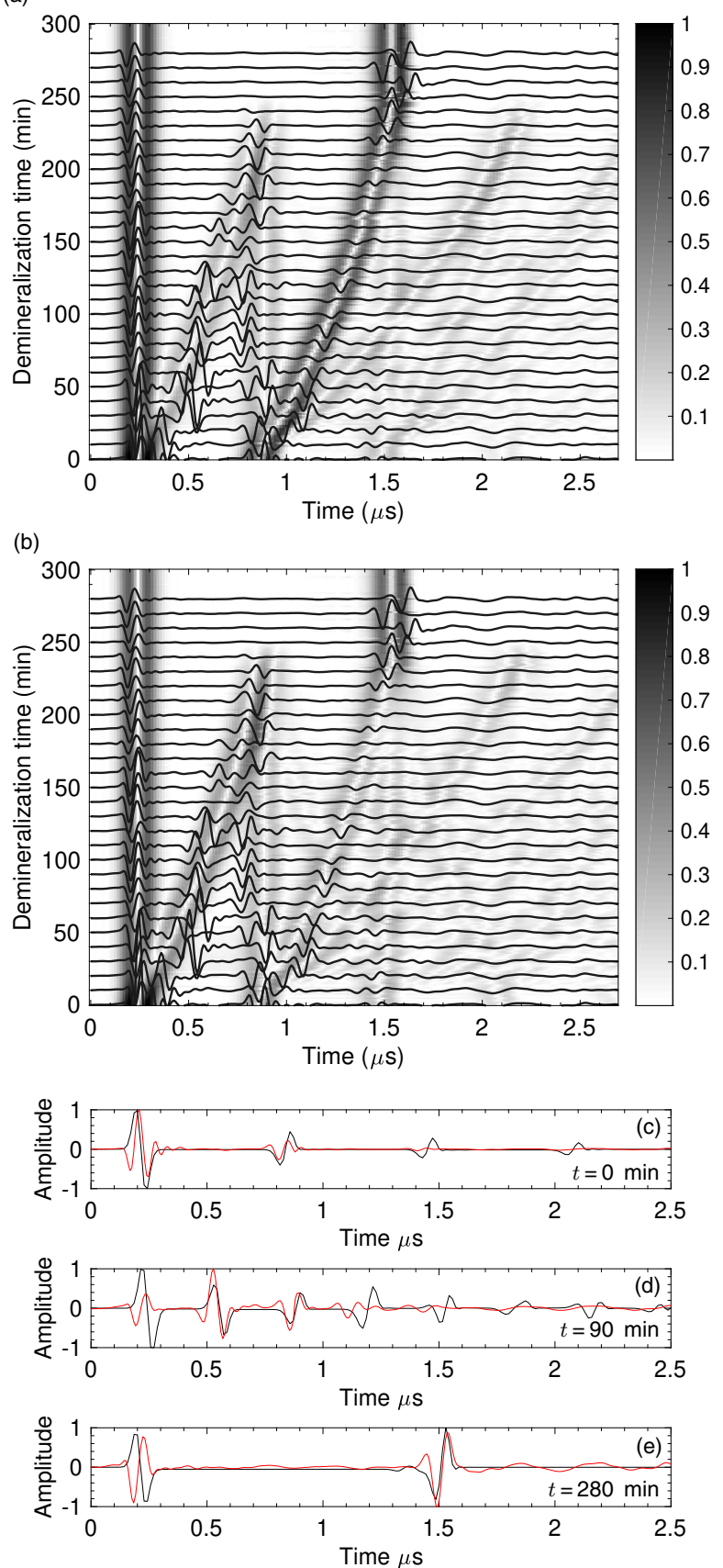

Fig. 6. RF-signals of sample D1 obtained experimentally (lines) and using a FEM simulation (normalized, gray scale surface, equivalent to single-element M-mode images) for (a) a media with homogeneous density and layered sound speed, and (b) a layered media with changes in density and sound speed. (c-e) RF signals obtained experimentally (red) and by simulation (black) at three stages of demineralization: (c) at the initial stage $(t=0 \mathrm{~min})$, (d) during demineralization $(t=90 \mathrm{~min})$ and (e) at the end of the experiment $(t=280$ $\min )$.

acidic solution, cleaned with distilled water and ultrasonically monitored according to the procedure described in Sec. II-D. Then, the sample was conserved in distilled water and continuously monitored with a frequency of one hour.

Figure 7 shows the demineralized distance as a function of time for the 6 teeth samples. Error bars correspond to the standard deviation of 20 measurements. As it can be seen, 


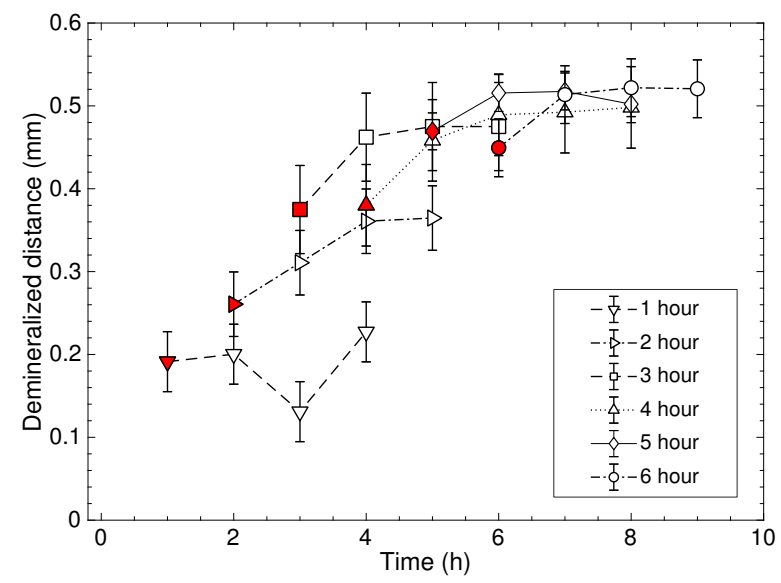

Fig. 7. First point in each set (red markers): Demineralized distance as a function of time maintained in acidic solution. Next points in each set (white markers): evolution of the demineralized distance once the samples have been removed from the acidic solution.

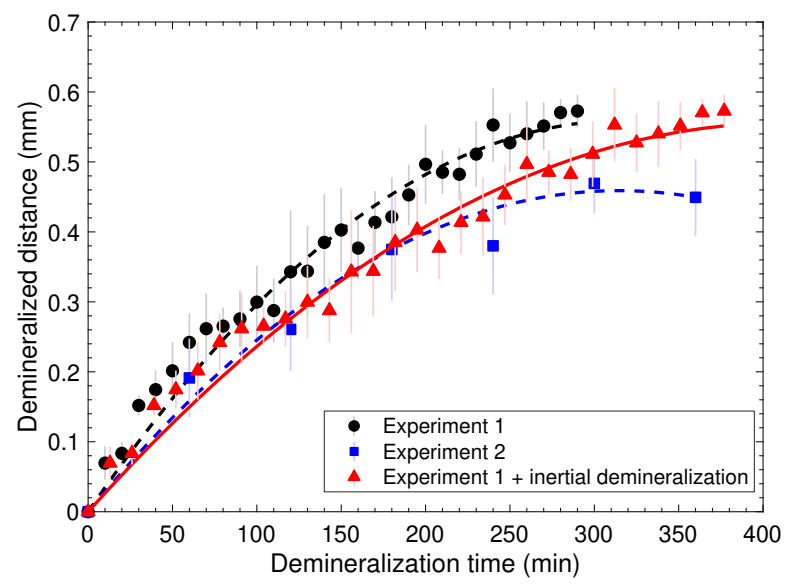

Fig. 8. Demineralized distance versus demineralization time in experiment E1 (black circles), experiment E2 (blue squares) and experiment E1 corrected using the inertial demineralization (red triangles). Lines shows the polynomial fits for E1 and E2 (dashed lines), and E1 with the inertial correction (red line).

the demineralized distance is higher the longer the dentin has been conserved in the acidic solution (first point in each curve), but it can also be observed (subsequent points in each curve) that the demineralized distance continues to grow for each sample even when the tooth has been extracted from the acidic solution and conserved in distilled water. This effect is caused by the presence of phosphoric acid molecules in their undissociated state into the subsurface region [36], dissociating as hydrogen ions and phosphate ions are provided to attack the mineral.

The inertia of the demineralizing effect of the acid is more important the longer the sample has remained in the acidic solution, as the concentration of phosphoric acid molecules in the dentin is higher. Table II shows the demineralization distance produced once each tooth has been removed from the acidic solution and conserved in distilled water. It increases from $32 \mu \mathrm{m}$ for the sample maintained 1 hour in the acidic solution to $114 \mu \mathrm{m}$ for the sample maintained 4 hours. Then, the inertia of the demineralization process stopped, indeed, teeth 5 and 6 were almost completely demineralized when they were extracted from the acidic solution.

According to this, the value of the acid demineralization velocity obtained in experiment 1 is overestimated, as there has not been considered as demineralization time the 3 minutes necessary to carry out the ultrasonic monitoring measurements made every 10 minutes. Figure 8 shows the results obtained from experiments 1 and 2 , and their corresponding fits to Eq. (2). Both, the velocity of the demineralization process and the acceleration are lower in experiment 2 than in experiment 1 , see Table III, as a consequence of the inertia in the action of the acid. A correction to take into account the inertial effect of the acid during the ultrasonic test was introduced in the data of experiment 1 by accounting for the duration of the ultrasonic test. The correct data (red markers) in Fig. 8 and its corresponding polynomial fit agree the demineralization process measured in experiment 2. After the correction, the retrieved initial velocity and acceleration of the inner boundary are closer to the values of experiment 2 (see Table III), showing that inertial demineralization should be considered.

\section{CONCLUSIONS}

A pulse-echo ultrasonic technique has been proposed to study the artificial demineralization process of human dentin using a solution of phosphoric acid at $10 \%$ concentration. The ultrasonic system included a delay line to separate the receiving echoes from the emitting pulses, as well as to reduce the monitored area in the dentin surface to $3.1 \mathrm{~mm}^{2}$.

The whole dentin demineralization process has been ultrasonically monitored, showing that the acid reduces the stiffness of the dentin, and consequently the longitudinal wave velocity on it, forming a layered structure with well-defined boundaries. As demineralization occurs under the action of the acid, these boundaries progressively grow in depth following a decelerated pattern. This model, which fits the experimental data, has been corroborated through simulations. We observed that the demineralization process is not constant, it was estimated that the velocity of penetration of the demineralized layer starts at $3.5 \mu \mathrm{m} / \mathrm{min}$, i.e. when the acid attacks the surface of the dentin, but as the acid penetrates this velocity is decelerated by $-11 \mathrm{~nm} / \mathrm{min}^{2}$. In addition, it has been demonstrated the fact that the demineralization process does not stop once the sample is removed from the acidic solution. Instead, several extra tens of microns of dentin are demineralized depending on the concentration of cations in the dentin. The correction of the effect of the demineralization inertia changes the dynamic of the demineralization process to $2.9 \mu \mathrm{m} / \mathrm{min}$ for the initial demineralization velocity and $-9.3 \mathrm{~nm} / \mathrm{min}^{2}$ for the deceleration.

TABLE II

INERTIAL DEMINERALIZATION DISTANCE AFTER REMOVING SAMPLE FROM THE ACIDIC SOLUTION.

\begin{tabular}{lcccccc}
\hline Sample & D1 & D2 & D3 & D4 & D5 & D6 \\
\hline Inertial demin. $(\mu \mathrm{m})$ & 36.0 & 104.0 & 100.2 & 118.1 & 48.2 & 72.5 \\
\hline
\end{tabular}


TABLE III

FITTED PARAMETERS FOR THE PHENOMENOLOGICAL MODEL GIVEN BY EQ. (2).

\begin{tabular}{lcc}
\hline & $\begin{array}{c}\text { Initial velocity } \\
v_{0}(\mu \mathrm{m} / \mathrm{min})\end{array}$ & $\begin{array}{c}\text { Acceleration } \\
a_{d}\left(\mathrm{~nm} / \mathrm{min}^{2}\right)\end{array}$ \\
\hline Experiment 1 (E1) & $3.5 \pm 0.4$ & $-11.0 \pm 3.0$ \\
Experiment 2 (E2) & $2.9 \pm 0.2$ & $-9.3 \pm 1.4$ \\
Experiment 1 (E1+ inertial correction) & $2.7 \pm 0.4$ & $-6.5 \pm 3.0$ \\
\hline
\end{tabular}

The technique provides an indeterminacy of $40 \mu \mathrm{m}$ in the calculation of the demineralized distance, including uncertainties due to irregularities in the preparation of the samples, in the estimation of the time of flight and in the measurement of the thickness of the sample during the ultrasonic measurement. It should be said here that the sample can be slightly compressed during the contact between the delay line of the probe, especially when demineralization is important and, therefore, the stiffness of the dentin is reduced.

The proposed technique can be used to monitor artificial standardized demineralization lesions at laboratory conditions, but also, because of the reduced dimension of the probe, to evaluate demineralization produced by natural caries invivo. It is worth to mention here that the measurement of the thickness of the sample is not required to evaluate the relevance of a demineralization process, as the change in the stiffness between the demineralized and mineralized tissue provides a clear echo whose time of flight informs about the demineralization depth if a previous database of sound velocities have been obtained.

\section{ACKNOWLEDGMENT}

This work has been developed within the framework of the IVIO-UPV Chair. We want to thank the support and advice of Dr. Joan Faus. This research has been supported by the Spanish Ministry of Science, Innovation and Universities (MICINN) through grant "Juan de la Cierva - Incorporación" (IJC2018-037897-I) and program "Proyectos I+D+i 2019" (PID2019-111436RB-C22) and by the Agència Valenciana de la Innovació through grant INNCON/2020/009. Action cofinanced by the European Union through the Programa Operativo del Fondo Europeo de Desarrollo Regional (FEDER) of the Comunitat Valenciana 2014-2020 (IDIFEDER/2018/022)

\section{REFERENCES}

[1] D. P. Catherine Le Galès-Camus, Poul Erik Petersen, "Who releases new report on global problem of oral diseases," World Health Organization, 2004.

[2] C. Longbottom, M.-C. Huysmans, N. Pitts, and M. Fontana, "Glossary of key terms," in Monographs in Oral Science. KARGER, 2009, pp. 209-216. [Online]. Available: https://www.ncbi.nlm.nih.gov/pubmed/ 19494688

[3] R. H. Selwitz, A. I. Ismail, and N. B. Pitts, "Dental caries," The Lancet, vol. 369, no. 9555, pp. 51-59, jan 2007. [Online]. Available: https://www.ncbi.nlm.nih.gov/pubmed/17208642

[4] M. Torabinejad and N. Chivian, "Clinical applications of mineral trioxide aggregate," Journal of Endodontics, vol. 25, no. 3, pp. 197-205, mar 1999. [Online]. Available: https://www.ncbi.nlm.nih.gov/pubmed/ 10321187

[5] L. Han and T. Okiji, "Bioactivity evaluation of three calcium silicatebased endodontic materials," International Endodontic Journal, vol. 46, no. 9 , pp. $808-814$, feb 2013
[6] M. G. Gandolfi, F. Siboni, T. Botero, M. Bossù, F. Riccitiello, and C. Prati, "Calcium silicate and calcium hydroxide materials for pulp capping: biointeractivity, porosity, solubility and bioactivity of current formulations," Journal of Applied Biomaterials \& Functional Materials, vol. 13, no. 1, pp. 0-0, sep 2014.

[7] I. A. Mjör, "Human coronal dentine: Structure and reactions," Oral Surgery, Oral Medicine, Oral Pathology, vol. 33, no. 5, pp. 810-823, may 1972. [Online]. Available: https://www.sciencedirect.com/science/ article/abs/pii/0030422072904513

[8] Y.-C. Chien, A. Burwell, K. Saeki, A. Fernandez-Martinez, M. Pugach, G. Nonomura, S. Habelitz, S. Ho, M. Rapozo-Hilo, J. Featherstone, S. Marshall, and G. Marshall, "Distinct decalcification process of dentin by different cariogenic organic acids: Kinetics, ultrastructure and mechanical properties," Archives of Oral Biology, vol. 63, pp. 93-105, mar 2016. [Online]. Available: https://www.sciencedirect.com/science/ article/pii/S0003996915300509

[9] K. Saeki, Y.-C. Chien, G. Nonomura, A. Chin, S. Habelitz, L. Gower, S. Marshall, and G. Marshall, "Recovery after PILP remineralization of dentin lesions created with two cariogenic acids," Archives of Oral Biology, vol. 82, pp. 194-202, oct 2017. [Online]. Available: https://www.sciencedirect.com/science/article/pii/S0003996917301826

[10] B. Li, X. Zhu, L. Ma, F. Wang, X. Liu, X. Yang, J. Zhou, J. Tan, D. H. Pashley, and F. R. Tay, "Selective demineralisation of dentine extrafibrillar minerals - a potential method to eliminate water-wet bonding in the etch-and-rinse technique," Journal of Dentistry, vol. 52, pp. 55-62, sep 2016. [Online]. Available: https://www.sciencedirect.com/science/article/pii/S030057121630135X

[11] J. Perdigão, "Dentin bonding as a function of dentin structure," Dental Clinics of North America, vol. 46, no. 2, pp. 277-301, apr 2002. [Online]. Available: https://www.sciencedirect.com/science/article/abs/ pii/S0011853201000088?via\%3Dihub

[12] J. Perdigão, P. Lambrechts, B. V. Meerbeek, Â. R. Tomé, G. Vanherle, and A. B. Lopes, "Morphological field emission-SEM study of the effect of six phosphoric acid etching agents on human dentin," Dental Materials, vol. 12, no. 4, pp. 262-271, jul 1996. [Online]. Available: https://www.sciencedirect.com/science/article/pii/S0109564196800339

[13] K. D. Jandt, "Atomic force microscopy of biomaterials surfaces and interfaces," Surface Science, vol. 491, no. 3, pp. 303-332, oct 2001.

[14] J. Kinney, M. Balooch, D. Haupt, S. Marshall, and G. Marshall, "Mineral distribution and dimensional changes in human dentin during demineralization," Journal of Dental Research, vol. 74, no. 5, pp. 1179-1184, may 1995. [Online]. Available: https://journals.sagepub. com/doi/abs/10.1177/00220345950740050601

[15] C. H. Wilder-Smith et al., "Quantification of dental erosions in patients with gerd using optical coherence tomography before and after doubleblind, randomized treatment with esomeprazole or placebo," The American journal of gastroenterology, vol. 104, no. 11, p. 2788, 2009.

[16] F. Herkströter, M. Witjes, J. Ruben, and J. Arends, "Time dependency of microhardness indentations in human and bovine dentine compared with human enamel (short communication)," Caries Research, vol. 23, no. 5, pp. 342-344, 1989.

[17] T. Attin and F. J. Wegehaupt, "Methods for assessment of dental erosion," in Monographs in Oral Science. S. KARGER AG, 2014, pp. 123-142. [Online]. Available: https://www.karger.com/Article/Abstract/ 360355

[18] G. Baum, I. Greenwood, S. Slawski, and R. Smirnow, "Observation of internal structures of teeth by ultrasonography," Science, vol. 139, no. 3554, pp. 495-496, feb 1963. [Online]. Available: https: //science.sciencemag.org/content/139/3554/495

[19] S. Ghorayeb, C. Bertoncini, and M. Hinders, "Ultrasonography in dentistry," IEEE Transactions on Ultrasonics, Ferroelectrics and Frequency Control, vol. 55, no. 6, pp. 1256-1266, jun 2008. [Online]. Available: https://ieeexplore.ieee.org/abstract/document/4536920

[20] J. Marotti, S. Heger, J. Tinschert, P. Tortamano, F. Chuembou, K. Radermacher, and S. Wolfart, "Recent advances of ultrasound imaging in dentistry - a review of the literature," Oral Surgery, Oral Medicine, Oral Pathology and Oral Radiology, vol. 115, no. 6, pp. 819-832, jun 2013. [Online]. Available: https://www.sciencedirect.com/ science/article/pii/S2212440313001727

[21] T. Watanabe, M. Miyazaki, H. Inage, and H. Kurokawa, "Determination of elastic modulus of the components at dentin-resin interface using the ultrasonic device," Dental Materials Journal, vol. 23, no. 3, pp. 361-367, 2004. [Online]. Available: https://www.jstage.jst.go.jp/article/ dmj1982/23/3/23_3_361/_article/-char/ja/

[22] G. Yasuda, H. Inage, T. Takamizawa, H. Kurokawa, A. Rikuta, and M. Miyazaki, "Determination of elastic modulus of demineralized resin-infiltrated dentin by self-etch adhesives," European Journal of 
Oral Sciences, vol. 115, no. 1, pp. 87-91, feb 2007. [Online]. Available: https://www.ncbi.nlm.nih.gov/pubmed/17305722

[23] S. Ng, M. Ferguson, P. Payne, and P. Slater, "Ultrasonic studies of unblemished and artificially demineralized enamel in extracted human teeth: a new method for detecting early caries," Journal of Dentistry, vol. 16, no. 5, pp. 201-209, oct 1988. [Online]. Available: https://www.sciencedirect.com/science/article/pii/030057128890070X

[24] S. Ng, P. Payne, N. Cartledge, and M. Ferguson, "Determination of ultrasonic velocity in human enamel and dentine," Archives of Oral Biology, vol. 34, no. 5, pp. 341-345, 1989. [Online]. Available: https://www.sciencedirect.com/science/article/pii/0003996989901076

[25] R. G. Maev, L. A. Denisova, E. Y. Maeva, and A. A. Denissov, "New data on histology and physico-mechanical properties of human tooth tissue obtained with acoustic microscopy," Ultrasound in Medicine \& Biology, vol. 28, no. 1, pp. 131-136, jan 2002. [Online]. Available: https: //www.sciencedirect.com/science/article/abs/pii/S030156290100480X

[26] M. Miyazaki, H. Inage, and H. Onose, "Use of an ultrasonic device for the determination of elastic modulus of dentin." Journal of Oral Science, vol. 44, no. 1, pp. 19-26, 2002. [Online]. Available: https:// www.jstage.jst.go.jp/article/josnusd1998/44/1/44_1_19/_article/-char/ja/

[27] C. John, "The corono-apically varying ultrasonic velocity in human hard dental tissues," The Journal of the Acoustical Society of America, vol. 116, no. 1, pp. 545-556, jul 2004.

[28] _ , "The laterally varying ultrasonic velocity in the dentin of human teeth," Journal of Biomechanics, vol. 39, no. 13, pp. 2388-2396, jan 2006.

[29] C. Lost, K.-M. Irion, C. John, and W. Nussle, "Two-dimensional distribution of sound velocity in ground sections of dentin," Dental Traumatology, vol. 8, no. 5, pp. 215-218, oct 1992. [Online] Available: https://onlinelibrary.wiley.com/doi/abs/10.1111/j.1600-9657. 1992.tb00246.x

[30] C. John, K. Irion, W. Nüssle, and C. Löst, "The resolution of a 2-dimensional ultrasonic velocity profile of human tooth sections," Schweizer Monatsschrift fur Zahnmedizin= Revue mensuelle suisse d'odonto-stomatologie $=$ Rivista mensile svizzera di odontologia e stomatologia, vol. 104, no. 1, pp. 25-30, 1994. [Online]. Available: https://europepmc.org/article/med/8108688

[31] S. Lees, F. Gerhard, and F. Oppenheim, "Ultrasonic measurement of dental enamel demineralization," Ultrasonics, vol. 11, no. 6, pp. 269-273, nov 1973. [Online]. Available: https://www.sciencedirect.com/ science/article/abs/pii/0041624X73901042

[32] K. Yamaguchi, M. Miyazaki, T. Takamizawa, H. Inage, and B. K. Moore, "Effect of CPP-ACP paste on mechanical properties of bovine enamel as determined by an ultrasonic device," Journal of Dentistry, vol. 34, no. 3, pp. 230-236, mar 2006. [Online]. Available: https://www.ncbi.nlm.nih.gov/pubmed/16112336

[33] K. Yamaguchi, M. Miyazaki, T. Takamizawa, H. Inage, and H. Kurokawa, "Ultrasonic determination of the effect of casein phosphopeptide-amorphous calcium phosphate paste on the demineralization of bovine dentin," Caries Research, vol. 41, no. 3, pp. 204-207, 2007. [Online]. Available: https://www.karger.com/Article/Abstract/99319

[34] H. Endo, R. Kawamoto, F. Takahashi, H. Takenaka, F. Yoshida, K. Nojiri, T. Takamizawa, and M. Miyazaki, "Evaluation of a calcium phosphate desensitizer using an ultrasonic device," Dental Materials Journal, vol. 32, no. 3, pp. 456-461, 2013. [Online]. Available: https://www.ncbi.nlm.nih.gov/pubmed/23719008

[35] R. Carvalho, M. Yoshiyama, E. Pashley, and D. H. Pashley, "In vitro study on the dimensional changes of human dentine after demineralization," Archives of oral biology, vol. 41, no. 4, pp. 369-377, 1996.

[36] J. Featherstone and A. Lussi, "Understanding the chemistry of dental erosion," in Monographs in Oral Science. KARGER, 2006, pp. 66-76.

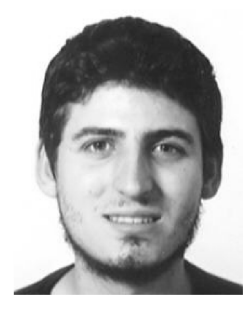

Josep Rodríguez-Sendra. BSc in Telecommunication (2014) and MSc in Acoustics (2015) by the Universitat Politècnica de València (UPV). In 2016 he enrolled the team Ultrasound Medical and Industrial Laboratory (UMIL) at I3M to research on Ultrasonic technology applied to odontology. Since 2017 he is $\mathrm{PhD}$ student at Instituto de Instrumentación para Imagen Molecular (I3M). His research interest concerns new technologies for biomedical ultrasound applications.

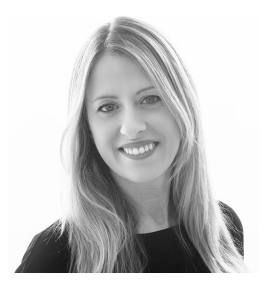

Inés Torres. DDS by Cardenal Herrera CEU University in 2008. Postgraduated programs in Endodontics (2009), Orthodontics (2011) and dental prosthetics (2013). Associate professor of Dental Pathology and Therapy at Cardenal Herrera-CEU since 2009. Co-director of Endodontic postgraduated program at IVIO (Instituto Valenciano de Investigaciones Odontológicas) since 2016. She collaborates also in other postgraduated dental programs. Dental private practice exclusively to endodontics. Actually, she's studying a PhD program based on Dental Biomaterials. Member of "Asociación Española de Endodoncia".

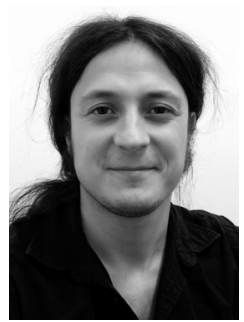

Noé Jiménez. BSc in Telecommunication, MSc in Acoustics and Ph.D in Acoustics by the Universitat Politècnica de València in 2015. In 2014 and 2019 he worked for the European Space Agency for noise control at the launch pad using periodic structures. In 2015 he joined the French CNRS (UMR6613) for a post-doctoral position to research on deepsubwavelength metamaterials. In 2017 he enrolled the Spanish National Research Council (CSIC) to research on biomedical ultrasound applications at Instituto de Instrumentación para Imagen Molecular (I3M). His research interests concern from fundamental research in waves in complex and structured media to biomedical ultrasound applications. Since 2013 he has published 38 journal papers and participated in more than 100 conferences. He has been visiting researcher at Columbia University (NY, USA) and at the University of Salford (Manchester, UK).

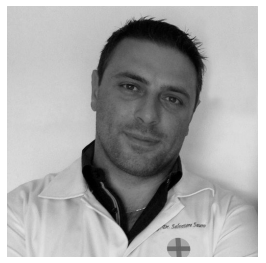

Salvatore Sauro. Adjunct Professor (Senior lecture full-time) Dental Biomaterials and Minimally Invasive Dentistry, University CEU-Cardenal Herrera, Valencia, Spain. Honorary senior lecturer, Biomaterials, Biophotonics and Tissue Engineering, King's College London Dental Institute (KCLDI) at Guy's Hospital, London (UK). Visiting Professor, Federal University of Moscow, School of Dentistry, Moscow, Russia and at the University of Hong Kong, Dental School, China. Dr. Sauro has been working in dental biomaterials, preventive and minimally invasive dentistry research for 15 years (JCR - H-Index: 31 ) and he has published, in collaboration with internationally renowned researchers, more than 120 articles in international peer-review journals with high impact in the dental and biomaterials field. 


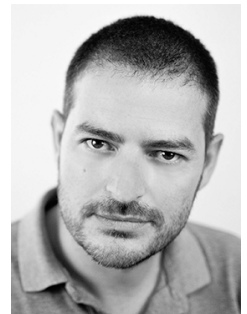

Francisco Camarena. Ph.D. in physics by the Universitat de València in 2003. He is a Founder Member and is the Head of the Ultrasound Medical and Industrial Laboratory (UMIL). He is a permanent researcher at Instituto de Instrumentación para Imagen Molecular (i3M), and associate professor in the Department of Applied Physics, UPV. He has participated in more than 80 national and international conferences related to acoustics and ultrasonics. He has authored or coauthored around 60 articles in national and international peer-reviewed journals. Dr. Camarena is the Head of the IVIO Chair (Instituto Valenciano de Investigaciones Odontológicas) IVIO-UPV, dedicated to the promotion and development of training activities, research, dissemination, and technology transfer in the field of odontology. He is the Director of the Scientific Unit of Business Innovation (UCIE, i3M). 